\title{
Lesões traumáticas de nervos cranianos
}

\author{
Luiz Fernando Cannoni ${ }^{1}$, Luciano Haddad ${ }^{1}$, Nelson Saade ${ }^{1}$, \\ Márcio Alexandre Teixeira da Costa ${ }^{2}$, José Carlos Esteves Veiga ${ }^{3}$
}

\section{RESUMO}

Objetivo: Documentar a incidência de lesões traumáticas de nervos cranianos, assim como a etiologia traumática, correlacionar as lesões dos nervos cranianos com achados radiológicos (lesões cranianas e intracranianas) e estudar lesões múltiplas de nervos cranianos. Métodos: Cinquenta e quatro pacientes admitidos no Serviço de Emergência da Santa Casa de Misericórdia de São Paulo com lesões traumáticas nos diferentes nervos cranianos foram incluídos. Todos os pacientes foram submetidos à radiografia simples de crânio, tomografia computadorizada e, quando indicada, ressonância magnética de encéfalo. Os pacientes foram divididos em três grupos de acordo com o escore da Escala de Coma de Glasgow (ECG) em: trauma leve (ECG de 13 a 15), moderado (ECG de 9 a 12) e grave (ECG de 3 a 8). Resultados: Os nervos cranianos mais afetados de forma isolada foram olfatório, facial e oculomotor. O atropelamento foi a causa mais comum de lesão de nervos cranianos de forma isolada, assim como nas lesões de múltiplos nervos. Contusões e hematomas extradurais foram as lesões intracranianas mais frequentes. Conclusão: Neuropatia craniana de etiologia traumática ocorre frequentemente e deve ser pesquisada à admissão do paciente, pois pode exigir descompressão de estruturas nervosas importantes como nervo óptico e facial.

\section{PALAVRAS-CHAVE}

Traumatismos dos nervos cranianos, traumatismos craniocerebrais, traumatismos do nervo óptico.

\section{ABSTRACT}

Traumatic lesions to the cranial nerves

Objective: To register the incidence of the traumatic lesions to the cranial nerves and its etiology; to correlate the lesions to the radiological findings (cranial and intracranial) and study multiple cranial nerve lesions. Methods: Fifty-four patients admitted to the Emergency Service of Santa Casa de Misericórdia de São Paulo Hospital have been studied and lesions to the different cranial nerves were described. All patients were submitted do radiographic exams, computed tomography, and, when necessary, magnetic resonance imaging. The patients were divided into 3 groups according to the Glasgow Coma Scale (GCS) in: mild trauma (GCS: 13 to 15), moderate (GCS: 9 to 12) and severe (GCS: 3 to 8). Results: Posttraumatic single nerve lesion was more frequent seen on olfactory, facial and oculomotor nerves. Running over was the main cause of these lesions (single nerve and multiple nerves). Contusions and extradural hematomas were the most frequent intracranial lesions. Conclusion: Traumatic cranial neuropathy occurs frequently and must be searched on the patient admission, because it can surgical decompression may necessary, such as decompression of the optic or facial nerves.

\section{KEYWORDS}

Cranial nerve injuries, craniocerebral trauma, optic nerve injuries.

1 Médico-assistente da disciplina de Neurocirurgia da Irmandade Santa Casa de Misericórdia de São Paulo (ISCMSP), São Paulo, SP, Brasil.

2 Médico-residente da disciplina de Neurocirurgia da ISCMSP, São Paulo, SP, Brasil.

3 Professor adjunto e chefe da disciplina de Neurocirurgia da ISCMSP, São Paulo, SP, Brasil. 


\section{Introdução}

O aumento progressivo no número de pacientes vítimas de traumatismo craniencefálico com consequentes lesões a nervos cranianos e a escassez de estudos nessa área fazem com que sejam necessários a elaboração de protocolos e o seguimento deles em nível ambulatorial para proposta de tratamento. A lesão traumática de nervos cranianos (penetrante ou não) é frequente. De acordo com Patel et al., ${ }^{1}$ em 12,6\% dos casos de sua série ocorreram lesões dos diferentes nervos cranianos. São lesões frequentemente negligenciadas nos exames neurológicos de entrada em prontos-socorros e muitas vezes somente perceptíveis ou evidentes tardiamente durante a evolução do processo traumático. Pacientes comatosos podem ter lesões traumáticas de nervos cranianos e, por causa do comprometimento do nível de consciência, estas podem não ser detectadas ao exame inicial. Exceções fazem-se aos nervos oculomotor (III), abducente (VI) e facial (VII), em que podem ser detectadas lesões mesmo com o paciente inconsciente.

Existem algumas "armadilhas" quanto ao diagnóstico de lesão de nervos cranianos: a perda de olfação pode ocorrer por simples obstrução nasal, e não por lesão própria em nervo olfatório; bloqueio do canal auditivo externo ou lesão da orelha média podem apresentar diminuição da acuidade auditiva sem lesão do oitavo nervo.

Por esses motivos, deve-se estar muito atento ao paciente que apresenta agudamente determinada lesão ou que a desenvolve tardiamente. Deve-se considerar ainda o paciente que apresenta lesão prévia de determinado nervo, seja por doença sistêmica como diabetes mellitus, trauma prévio ou cirurgias cranianas, orbitárias, faciais e plásticas.

Como primeiro relato de lesão traumática de nervo craniano, cita-se Hipócrates ${ }^{1}$ quando descreve paciente com acometimento da acuidade visual após trauma em região frontal.

Patel et al., ${ }^{1}$ Keane e Baloh ${ }^{2}$ e Leigh ${ }^{3}$ descrevem que as lesões traumáticas são mais frequentes nos nervos olfatório (I), facial (VII) e vestíbulo-coclear (VIII), seguidas por lesões nos nervo óptico (II) e complexo oculomotor (III, IV e VI) e menos frequentemente o trigêmeo (V) e os nervos cranianos baixos (IX, X, XI e XII).

As lesões dos nervos cranianos podem sugerir $\mathrm{O}$ tipo de trauma que as causou. ${ }^{2}$ Em traumas penetrantes (tentativas de suicídio) com ferimento de entrada em região anterior da orelha, geralmente há transecção do quiasma óptico. Em fraturas da porção basilar do osso occipital (alta energia cinética), nervos cranianos inferiores são acometidos.

Os nervos cranianos fazem parte do sistema nervoso periférico e possuem componentes motores, sensitivos e autonômicos. Cursam por diferentes trajetos no interior do crânio e exteriorizam-se através dos forames cranianos localizados na órbita e na base do crânio. Esses forames são frequentemente envolvidos em traumas craniencefálicos (trauma direto), tornando a lesão de nervos cranianos frequente quando se analisam esses pacientes. Além disso, devem ser consideradas as lesões por estiramento e avulsão desses nervos quando do deslocamento cerebral (movimento de aceleração e desaceleração do encéfalo em relação ao crânio com lesão da dura-máter pela superfície anfractuosa da base do crânio). As lesões por estiramento ocorrem nas porções dos nervos fixas ao crânio ou em pontos de grande angulação do seu trajeto. Pode também haver lesão na condução nervosa por injúria sofrida ao suprimento sanguíneo do nervo. ${ }^{4}$

\section{Casuística e método}

Estudaram-se 54 pacientes com lesões traumáticas de nervos cranianos atendidos no Pronto-Socorro Central da Santa Casa de Misericórdia de São Paulo no período de junho de 2010 a julho de 2012, por meio de estudo prospectivo baseado em protocolo próprio, previamente autorizado pelo Comitê de Ética daquela instituição.

Foram excluídos do estudo indivíduos portadores de lesões traumáticas prévias em nervos cranianos, assim como lesões por diabetes mellitus, esclerose múltipla, neoplasias primárias e secundárias e sífilis.

Todos os pacientes foram submetidos a exame neurológico e exames de imagem, que constaram de radiografia simples de crânio (100\%), tomografia computadorizada de crânio (100\%), ressonância nuclear magnética de encéfalo (10\%) e angiografia cerebral digital (10\%) no momento da admissão e repetição deles quando indicado.

O exame neurológico dos nervos cranianos foi feito à admissão, durante a internação e durante o acompanhamento ambulatorial.

Como critérios de lesões, incluíram-se os seguintes aspectos para cada nervo craniano:

a) nervo olfatório: hiposmia uni ou bilateral, anosmia uni ou bilateral (café e canela);

b) nervo óptico: diminuição da acuidade visual ou do campo visual por confrontação direta, campimetria ou diagnosticados pela disciplina de Oftalmologia;

c) nervo oculomotor: alteração na motricidade ocular extrínseca e intrínseca, queda palpebral;

d) nervo troclear: alteração da motricidade ocular extrínseca; 
e) nervo trigêmeo: alteração de sensibilidade facial correspondente ao território de inervação de qualquer dos três ramos principais ou de suas divisões ou alteração da motricidade da musculatura mastigatória;

f) nervo abducente: alteração da motricidade ocular extrínseca;

g) nervo facial: alteração da motricidade da musculatura facial segundo a escala de House-Brackmann ou alteração gustativa dos $2 / 3$ anteriores da língua.

h) nervo vestíbulo-coclear: diminuição da acuidade auditiva diagnosticada por diapasão, audiometria ou avaliação da disciplina de Otorrinolaringologia;

i) nervo glossofaríngeo: alteração da musculatura da deglutição e do véu palatino;

j) nervo vago: distúrbios da fonação avaliados pela equipe de fonoaudiologia;

k) nervo acessório: alteração da motricidade dos músculos esternoclidomastóideo e trapézio;

1) nervo hipoglosso: alteração da movimentação da língua.

Os pacientes foram seguidos em âmbito ambulatorial em períodos regulares e avaliados sempre pelo autor (LFC) durante o primeiro, terceiro, sexto e décimo segundo meses após a lesão.

\begin{tabular}{|c|c|}
\hline \multicolumn{2}{|c|}{$\begin{array}{l}\text { Tabela } 1 \text { - Incidência de lesões traumáticas em } \\
\text { nervos cranianos (nervos isoladamente lesados) }\end{array}$} \\
\hline Nervo & Lesões isoladas \\
\hline Olfatório & $8(29,63 \%)$ \\
\hline Óptico & $1(3,70 \%)$ \\
\hline Oculomotor & $6(22,22 \%)$ \\
\hline Troclear & $1(3,70 \%)$ \\
\hline Trigêmeo & $0(0,00 \%)$ \\
\hline Abducente & $1(3,70 \%)$ \\
\hline Facial & $7(25,93 \%)$ \\
\hline Vestíbulo-coclear & $3(11,11 \%)$ \\
\hline Total & 27 casos \\
\hline
\end{tabular}

\section{Resultados}

Dos 54 pacientes admitidos, 30 (55,56\%) obtiveram escore de 13 a 15 na Escala de Coma de Glasgow à admissão e foram considerados como portadores de trauma craniano leve; o trauma moderado (Glasgow 9 a 12) foi atribuído a 9 pacientes $(16,67 \%)$ e o trauma craniano grave (Glasgow 3 a 8), a 15 pacientes (27,78\%).

O traumatismo craniencefálico é mais comum no gênero masculino. Em nosso estudo, observou-se o ní- tido predomínio das lesões nesse gênero (41 pacientes representando $75,93 \%$ da casuística) e 13 no gênero feminino (24,07\%). A média de idade foi de 36,14 anos.

A seguir, apresentamos a casuística de nervos lesados isoladamente, (Tabela 1) em associação (lesões múltiplas), (Tabela 2) assim como a presença e a localização de fraturas cranianas, lesões intracranianas e mecanismo de trauma: (Tabela 3).

\begin{tabular}{|c|c|c|c|}
\hline \multicolumn{4}{|c|}{$\begin{array}{c}\text { Tabela } 2 \text { - Incidência de lesões traumáticas em nervos } \\
\text { cranianos (nervos lesados em associação) }\end{array}$} \\
\hline \multicolumn{2}{|l|}{ Nervo } & \multicolumn{2}{|c|}{ Lesões associadas } \\
\hline \multicolumn{2}{|l|}{ VII/VIII } & \multicolumn{2}{|c|}{$6(22,22 \%)$} \\
\hline \multicolumn{2}{|l|}{$\mathrm{I} / \mathrm{II}$} & \multicolumn{2}{|c|}{$3(11,11 \%)$} \\
\hline \multicolumn{2}{|l|}{ I/VIII } & \multicolumn{2}{|c|}{$3(11,11 \%)$} \\
\hline \multicolumn{2}{|l|}{$\mathrm{I} / \mathrm{II} / \mathrm{V}$} & \multicolumn{2}{|c|}{$2(7,41 \%)$} \\
\hline \multicolumn{2}{|l|}{ III/VII } & \multicolumn{2}{|c|}{$1(3,70 \%)$} \\
\hline \multicolumn{2}{|l|}{ I/II/III/V } & \multicolumn{2}{|c|}{$1(3,70 \%)$} \\
\hline \multicolumn{2}{|c|}{ I/II/III/V/VI/VII/VIII } & \multicolumn{2}{|c|}{$1(3,70 \%)$} \\
\hline \multicolumn{2}{|l|}{ I/V/VI } & \multicolumn{2}{|c|}{$1(3,70 \%)$} \\
\hline \multicolumn{2}{|l|}{ II/III } & \multicolumn{2}{|c|}{$1(3,70 \%)$} \\
\hline \multicolumn{2}{|l|}{ II/III/IV } & \multicolumn{2}{|c|}{$1(3,70 \%)$} \\
\hline \multicolumn{2}{|l|}{ II/III/IV/V/VI } & \multicolumn{2}{|c|}{$1(3,70 \%)$} \\
\hline \multicolumn{2}{|c|}{ II/III/V/VI/VII } & \multicolumn{2}{|c|}{$1(3,70 \%)$} \\
\hline \multicolumn{2}{|c|}{ II/III/VI/VII/VIII } & \multicolumn{2}{|c|}{$1(3,70 \%)$} \\
\hline \multicolumn{2}{|l|}{ III/V } & \multicolumn{2}{|c|}{$1(3,70 \%)$} \\
\hline \multicolumn{2}{|l|}{ III/VIII } & $1(3$, & $0 \%)$ \\
\hline V/VII & & $1(3$, & $0 \%)$ \\
\hline VI/VIII & & $1(3$, & $0 \%)$ \\
\hline Total & & $27 c$ & $\operatorname{sos}$ \\
\hline $\begin{array}{l}\text { I: nervo olfatório; } \\
\text { trigêmeo; VI: ner }\end{array}$ & $\begin{array}{l}\text { rvo óptico; III: ner } \\
\text { ducente; VII: nervo }\end{array}$ & $\begin{array}{l}\text { culomotor; IV: nervo } \\
\text { ial; VIII: nervo vestib }\end{array}$ & $\begin{array}{l}\text { troclear; V: nervo } \\
\text { lo-coclear. }\end{array}$ \\
\hline $\begin{array}{r}\text { Tabel } \\
r e \\
\text { intro }\end{array}$ & $\begin{array}{l}\text { - Lesões trauı } \\
\text { onadas à pres } \\
\text { nianas associ }\end{array}$ & $\begin{array}{l}\text { ticas de nervos } \\
\text { ça de fraturas, } \\
\text { is e etiologia tra }\end{array}$ & $\begin{array}{l}\text { ranianos } \\
\text { esões } \\
\text { ımática }\end{array}$ \\
\hline Nervo & Fratura & $\begin{array}{l}\text { Lesões } \\
\text { intracranianas }\end{array}$ & $\begin{array}{l}\text { Etiologia } \\
\text { traumática }\end{array}$ \\
\hline Olfatório & Parietal & HED & Queda de altura \\
\hline Óptico & Teto de órbita & Contusão & $\begin{array}{l}\text { Acidente } \\
\text { motociclístico }\end{array}$ \\
\hline Oculomotor & Teto de órbita & $\begin{array}{l}\text { Contusão } \\
\text { e HSAt }\end{array}$ & $\begin{array}{l}\text { Acidente } \\
\text { motociclístico }\end{array}$ \\
\hline Troclear & Frontal & Ausente & $\begin{array}{l}\text { Acidente } \\
\text { motociclístico e } \\
\text { atropelamento }\end{array}$ \\
\hline Trigêmeo & Teto de órbita & HED/HSAt & $\begin{array}{l}\text { Acidente } \\
\text { motociclístico }\end{array}$ \\
\hline Abducente & Temporal & HED & $\begin{array}{l}\text { Acidente } \\
\text { motociclístico }\end{array}$ \\
\hline Facial & $\begin{array}{l}\text { Temporal e } \\
\text { petrosa }\end{array}$ & Contusão & $\begin{array}{l}\text { Acidente } \\
\text { motociclístico }\end{array}$ \\
\hline $\begin{array}{l}\text { Vestíbulo- } \\
\text { coclear }\end{array}$ & $\begin{array}{l}\text { Temporal e } \\
\text { petrosa }\end{array}$ & HED e contusão & $\begin{array}{l}\text { Acidente } \\
\text { automobilístico }\end{array}$ \\
\hline
\end{tabular}

HED: hematoma extradural, HSAt: hemorragia subaracnoide traumática. 


\section{Discussão}

Os nervos mais comumente afetados de forma isolada foram o olfatório $(29,63 \%)$, facial $(25,93 \%)$ e oculomotor (22,22\%). Quando mais de um nervo foi lesado, a associação mais comum foi a lesão do facial com o vestíbulo-coclear (seis casos). Não encontramos lesões pós-traumática em glossofaríngeo, vago, acessório e hipoglosso.

Nas lesões traumáticas dos nervos cranianos, a biomecânica do trauma está diretamente ligada ao prognóstico de recuperação funcional do nervo.

Além de serem lesados diretamente pelo trauma, podem ser lesados por reação tecidual presente em fraturas, aumento na pressão intracraniana e meningite.

\section{Nervo olfatório}

A anosmia ${ }^{5-7}$ é mais comum no trauma occipital, quando comparado ao trauma frontal. Há recuperação funcional do nervo em aproximadamente um terço dos casos nos primeiros três meses após o trauma. ${ }^{2} \mathrm{~A}$ frequência da anosmia é proporcional à intensidade do trauma.

Um teste importante para determinar a veracidade das afirmações do paciente no tocante à perda olfativa é verificar a integridade do nervo trigêmeo com substâncias aromáticas voláteis. Na integridade do trigêmeo, o indivíduo será capaz de reconhecê-las, assim como identificar substâncias ácidas (vinagre) quando em contato com a língua.

Embora a anosmia possa parecer completa, os pacientes relatam a preservação do paladar, que depende muito do aroma. Isso pode ser explicado nos casos de perda parcial da olfação por meio de aromas surgidos na nasofaringe.

O prognóstico é reservado nas lesões completas. ${ }^{5}$ Pode haver recuperação espontânea da olfação, ${ }^{8}$ mesmo que parcial, o que pode ser explicado pela ausência de lesão total do nervo e diminuição do processo edematoso, que frequentemente ocorre na fase aguda do trauma.

As alterações gustativas pós-traumáticas (hipogeusia e disgeusia) têm recebido muito menos atenção e ocorrem menos frequentemente em relação às alterações olfativas; a incidência de lesão chega até $4 \%$ dos pacientes. ${ }^{9-11}$ Evidenciaram-se alterações gustativas em dois casos com lesão exclusiva de nervo olfatório.

Geralmente as alterações gustativas acompanham as alterações olfativas, e na maioria dos casos regridem espontaneamente. Não há relação entre a severidade do trauma e o aparecimento de lesões gustativas. ${ }^{9}$

A etiologia da disgeusia ou ageusia pós-traumática é obscura. Sugere-se que sejam devidas à irritação ou à destruição do nervo olfatório. Leigh ${ }^{3}$ sugere que represente um estágio de recuperação da anosmia. Deve-se observar que alguns casos de alterações gustativas podem ocorrer na ausência de alterações olfativas. ${ }^{9}$

\section{Nervo óptico}

O nervo óptico possui aproximadamente $50 \mathrm{~mm}$ de comprimento e estende-se da porção posterior do globo ocular até o quiasma óptico, sendo essa uma divisão puramente funcional., ${ }^{2,12}$ Anatomicamente é dividido em quatro partes: intraocular, intraorbitária, intracanalicular e intracraniana.

Classifica-se a lesão traumática do nervo óptico em direta e indireta. A indireta resulta de trauma ipsilateral à região frontal..$^{13}$ Forças aqui aplicadas transmitem-se ao canal óptico, local mais frequente de lesão traumática do nervo óptico. ${ }^{14} \mathrm{O}$ trauma temporal e o parietal podem ocasionalmente causar lesão no nervo, e em raríssimas ocasiões o trauma occipital é a causa. Geralmente causam perda visual imediata e esta apresenta algum grau de recuperação em $33 \%$ dos casos. ${ }^{2} \mathrm{O}$ trauma direto aplica-se sobre o globo ocular, com avulsão do nervo óptico, associada à hemorragia retiniana.

O exame de fundo de olho pode não apresentar lesões agudas (palidez óptica ocorre após um mês do trauma), mas a ocorrência de hemorragias retinianas pode ser visualizada, assim como hemorragias vítreas. ${ }^{14}$

A análise radiológica pode evidenciar fraturas que envolvam o canal óptico. Devemos considerar que as hemorragias subaracnoides (mesmo as de origem traumática) podem cursar com hemorragias na bainha de mielina do nervo óptico e constituir causa de diminuição de acuidade visual, assim como ser acompanhadas de hemorragia vítrea (síndrome de Terson).

Cegueira unilateral é a complicação mais frequente da lesão traumática do nervo óptico, porém qualquer grau de acometimento da acuidade visual pode estar presente. No campo visual, os defeitos altitudinais inferiores são os mais frequentemente relatados., ${ }^{2,15}$

Deterioração progressiva da acuidade visual sugere processo de reversibilidade ${ }^{16}$ como hemorragia extra-axial ou compressão direta do nervo por fragmentos ósseos. Mesmo um curto período de visão após trauma craniano é, potencialmente, processo reversível. Por outro lado, perda visual imediata no impacto sugere laceração, avulsão ou contusão severa com necrose isquêmica do nervo e prognóstico muito reservado.

Em 10\% dos casos, há lesões bilaterais ou lesões quiasmáticas, ${ }^{17-19}$ que geralmente são assimétricas com comprometimento unilateral severo associadas a hemianopsia temporal contralateral. ${ }^{2}$ 
Embora muitos traumas de crânio envolvam a região frontal com fraturas de órbita, dano ao globo ocular é relativamente raro, pela própria proteção óssea da órbita e pela motilidade do globo ocular.

As lesões do globo ocular ocorrem frequentemente por trauma direto ou traumas penetrantes com consequente rotura do globo ocular. Nesses casos, geralmente o dano cerebral é mínimo, com excelente prognóstico, porém o dano ao globo ocular é uma importante fonte de infecção do sistema nervoso central, ${ }^{4}$ pela presença de fístula liquórica.

Deslocamento do eixo do olho ocorre durante o trauma quando a órbita é deformada por fraturas que envolvam a face ou a fronte. ${ }^{4}$ Os deslocamentos oculares mais frequentes ocorrem nas direções póstero-anteriores e desvio lateral do globo ocular. Deslocamentos no eixo superior e medial são raros. Consequentemente, a diplopia é queixa comum quando os neuromecanismos oculares estão lesados. Deve-se também considerar que a polia do músculo oblíquo superior contribui para os distúrbios do movimento ocular.

Trauma direto ao nervo óptico está confinado a ferimentos por projéteis de arma de fogo ao crânio e raramente são vistos na prática civil.

A porção intracanalicular do nervo óptico é a porção mais lesada pelo trauma craniano fechado. A porção intraorbitária geralmente é poupada, em virtude de sua mobilidade no interior da órbita e da proteção oferecida pela gordura periorbitária e músculos extraoculares.

Não existe um consenso quanto ao tratamento da lesão traumática do nervo óptico, mas é imperativo que alguma forma de tratamento seja feito no paciente que apresente sintomas de início tardio.

Patel et al. ${ }^{1}$ descrevem 22 lesões traumáticas de nervo óptico com $100 \%$ de presença de fraturas orbitais. Fraturas orbitais múltiplas com extensão ao ápex foram seguidas de fraturas da parede lateral da órbita, parede medial e teto da órbita em ordem de frequência.

Esses autores, assim como Joseph et al., ${ }^{16}$ preconizam a cirurgia de descompressão do nervo óptico quando fragmentos ósseos comprimem o nervo óptico (baseado na tomografia de órbita) associada à administração de altas doses de corticoides (metilprednisolona - $1 \mathrm{a} 2 \mathrm{~g}$ ), obtendo resultados de pobre melhora da acuidade visual.

Acredita-se que a infusão do corticoide reduza o edema no tecido lesado e previna a degeneração celular, ${ }^{20-23}$ além de aumentar o fluxo sanguíneo da área lesada, diminuindo o dano celular secundário à isquemia e à hipoxemia.

De Vries-Knoppert ${ }^{24}$ relata avulsão de nervo óptico como evento traumático raro que pode resultar de trauma facial ou orbitário, penetrante ou não. A avulsão é definida como separação do nervo óptico do globo ocular sem rotura da bainha do nervo ou da esclera adjacente. $\mathrm{O}$ diagnóstico pode não ser feito na fase aguda pela presença de hemorragia vítrea. A lesão inclui neurônios do nervo óptico, enquanto a bainha que o envolve permanece íntegra. $\mathrm{O}$ exame de fundo de olho revela lesão escurecida na região do disco óptico, rodeado por hemorragia retiniana. $\mathrm{Na}$ fase crônica a hemorragia é substituída por tecido fibroso. Perda visual tardia também pode ocorrer e é potencialmente reversível. Ocorre por desenvolvimento de edema ou isquemia no interior do canal óptico ou compressão por hematoma subperiostal. ${ }^{2,25,26}$ Mais raramente ocorre por desenvolvimento de mucoceles ou aneurismas traumáticos.

De acordo com Guy et al., ${ }^{27}$ o exato mecanismo de perda visual por trauma craniano não penetrante é duvidoso e seu tratamento é controverso. Acredita-se que a lesão ocorra por espasmo vascular ou oclusão de vaso nutriente. Outras causas incluem avulsão ou contusão do nervo óptico, fratura craniana com compressão do nervo por fragmento ósseo, edema do nervo óptico com compressão intracanalicular e deformidade orbitária.

\section{Complexo oculomotor}

\section{a) Nervo oculomotor}

Trata-se de lesões comumente associadas ao trauma craniano em adultos. ${ }^{28,29} \mathrm{Em}$ crianças, descrevem-se lesões congênitas, neoplásicas, infecciosas, vasculares (aneurismas) e migrânia oftalmoplégica ${ }^{30}$ como causa de alteração na função do nervo oculomotor e o trauma constitui a causa adquirida mais frequente de lesão desse nervo, seguida de processos infecciosos do sistema nervoso central. ${ }^{31}$ São traumas com alto componente cinético, geralmente dirigido à região frontal com fratura craniana e perda da consciência.

Dilatação pupilar associada ao desvio lateral do globo ocular em paciente vítima de TCE faz o diagnóstico de lesão do nervo oculomotor mesmo no paciente inconsciente. ${ }^{32}$ Uma pupila midriática fixa após trauma facial ou orbitário geralmente representa lesão direta do nervo ou dano ao sistema nervoso autônomo parassimpático.

$\mathrm{Na}$ fase aguda, ptose e paralisia extraocular podem não ser devidamente diagnosticadas, exceto quando houver paralisia total do III nervo. ${ }^{33}$ Deve-se lembrar que alterações pupilares por lesão de III nervo podem ser causadas por trauma orbitário ou região temporoparietal (relação do III nervo com a porção petroclinoide do tentório, que pode ser estirado durante o trauma craniano). Portanto, é extremamente importante diferenciar o comprometimento primário (lesão direta) do 
III nervo da lesão secundária (consequente ao aumento de hematoma intracraniano).

Para distinguir lesão de III nervo da limitação de movimento orbitário por lesão muscular, geralmente é necessário esperar a redução do edema orbitário. ${ }^{2}$

\section{b) Nervo troclear}

Willbrand e Sanger, citados por Chapman et al. ${ }^{34}$ afirmam que o nervo troclear é frequentemente lesado no véu medular anterior no nível do colículo inferior, onde as fibras do troclear decussam.

Fraqueza do músculo oblíquo superior causa diplopia vertical, que piora quando o paciente olha para baixo. ${ }^{35,36}$ Para compensar isso, o paciente aprende a adotar determinada postura da cabeça. Para compensar os desvios horizontais, o paciente adota deslocamento lateral da cabeça, e para desvios verticais, adota elevação ou depressão do queixo. Para desvios torsionais, inclina lateralmente a cabeça. Assim, a lesão do músculo oblíquo superior direito faz com que o paciente vire sua cabeça para a esquerda e sua face para a direita e seu queixo para baixo, para facilitar a fusão de imagens e diminuir a diplopia.

A lesão ocorre após severo trauma craniencefálico, geralmente causado por acidente automobilístico ou motociclístico, ${ }^{37} \mathrm{em}$ que exista trauma orbitário direto ou frontal.

O IV nervo também é suscetível a lesões na região tentorial, o que é observado em traumas frontais oblíquos e não diretos, por movimento de aceleração e desaceleração. Lesões bilaterais do IV nervo também estão relacionadas a trauma de altíssima energia cinética que atinge diretamente a região frontal. ${ }^{38,39}$

O nervo troclear é o único nervo craniano que possui a totalidade de suas fibras cruzadas logo após emergirem do mesencéfalo. $O$ véu medular anterior é o local da decussação do IV nervo e aqui sua estrutura é extremamente fina e facilmente deslocada do mesencéfalo. Assim, trauma na região frontal causa transmissão das linhas de força que o lesam no véu medular anterior.

Após uma súbita desaceleração ou trauma em região frontal, o cérebro continua em movimento inercial contra o crânio em repouso. Isso leva a uma lesão em contragolpe na região mesencefálica, com laceração e rotura do nervo. Pela simetria das forças contundentes durante o trauma, pode haver lesão bilateral do nervo.

\section{c) Nervo abducente}

A lesão do VI nervo geralmente é causada por fraturas da porção petrosa do temporal, paralisia facial e surdez e, quando lesado isoladamente, possui o melhor prognóstico de recuperação. O déficit tardio geralmente decorre de hipertensão intracraniana ou meningite hemorrágica. ${ }^{2}$

Possui trajeto intracraniano longo desde a ponte até a órbita e está intimamente relacionado aos nervos trigêmeo e facial.

O nervo abducente é mais vulnerável no ponto em que passa inferiormente o ligamento petroesfenoidal (ligamento de Gruber).$^{40}$ Posterolateralmente a essa estrutura, encontra-se o cavo de Meckel, que abriga o gânglio trigeminal.

Nem todas as lesões do abducente resultam de fraturas. $\mathrm{O}$ nervo pode ser contundido, tracionado ou lacerado no momento do trauma. A laceração ou a rotura do nervo são raras e ocorrem quando a fratura envolve a pirâmide petrosa. ${ }^{41,42}$

Nos traumas de crânio, o nervo também pode ser lesado em traumas frontais, cujo impacto avulsiona o nervo contra o rígido ligamento petroclinoide. Um impacto de forte intensidade pode provocar um deslocamento encefálico no sentido craniocaudal e lesar o nervo abducente na incisura tentorial.

Fraturas que envolvem a fissura orbital superior tendem a lesar o abducente em conjunto com o oculomotor, troclear e trigêmeo.

Diplopia homônima resultante da paralisia do nervo abducente é achado frequente na clínica oftalmológica. É o achado objetivo do déficit na abdução ocular e pode significar vários tipos de doença oftalmológica. ${ }^{43}$

O prognóstico é bom (75\% de recuperação), ${ }^{4}$ apesar de funcionalmente poder haver reinervação anômala de grupos musculares com produção de movimentos anômalos do globo ocular e pobre reatividade pupilar.

Rucker $^{44}$ analisa 1.000 casos de lesão conjunta do complexo oculomotor e relata que em mais de $21 \%$ dos casos a causa não foi determinada. Em aproximadamente $14 \%$ de sua série a etiologia foi traumática, sendo o nervo abducente o mais frequentemente acometido (39,6\%), seguido pelo oculomotor $(24,5 \%)$ e pelo troclear (16,5\%). Quanto às lesões mistas, a lesão conjunta do oculomotor e troclear aparece na mesma frequência da lesão do oculomotor e abducente $(6,4 \%)$, seguida pela lesão dos três nervos conjuntamente $(5,7 \%)$ e finalmente pela lesão conjunta do troclear e abducente $(0,7 \%)$.

\section{d) Nervo trigêmeo}

A lesão traumática do nervo trigêmeo é rara ${ }^{45,46} \mathrm{e} a$ literatura referente a esse tipo de lesão é consequentemente escassa.

O gânglio trigeminal está intimamente relacionado ao osso temporal. Como suas três divisões partem da fossa média, estas são adjacentes ao ápice petroso.

Qualquer fratura que se estenda do temporal em direção ao clivus pode lesar o gânglio trigeminal ou 
qualquer de suas divisões. O nervo é particularmente vulnerável no ponto de angulação da raiz sensitiva, onde ultrapassa o forame dural em direção ao cavo de Meckel. Esse forame dural localiza-se na fossa posterior inferiormente à margem da pirâmide petrosa.

A primeira e segunda divisões podem ser lesadas quando o tronco encefálico desloca-se em movimento de desaceleração do crânio.

Mock apud Jefferson e Schorstein ${ }^{45}$ relata três casos de lesão traumática de nervo trigêmeo em 3.176 fraturas de crânio.

Lesões em ramos terminais do trigêmeo são mais frequentes. Russell ${ }^{47}$ relata 45 lesões traumáticas em nervos supraorbitários e oito lesões em infraorbitários em 1.000 casos de trauma craniencefálico.

Lesões em couro cabeludo, levando a anestesia e parestesias, geralmente são omitidas na literatura médica, uma vez que a atenção à lesão do nervo trigêmeo se faz ao seu gânglio sensitivo e de seus ramos intracranianos ou contidos no interior dos ossos faciais. Portanto, uma procura minuciosa dessas lesões sensitivas certamente trará aumento das casuísticas.

A lesão do componente sensitivo do nervo trigêmeo é frequente em traumas faciais. Deve-se à lesão de ramos sensitivos superficiais por ferimentos corto-contusos, contusões e fraturas.

O nervo oftálmico (V1) com suas ramificações (n. supraorbital e supratroclear) é frequentemente lesado em traumas que incidem na região supraorbitária. A lesão leva à fratura do seio frontal e etmoidal, com anestesia/hipoestesia na ponta do nariz ou na córnea.

$\mathrm{O}$ n. maxilar (V2) é a divisão mais frequentemente lesada no trauma, principalmente em fraturas do assoalho orbitário ou em traumas de altíssima energia cinética acompanhados de fratura do forame redondo. Descrita inicialmente por Charles Bell apud Jefferson e Schorstein, ${ }^{45}$ em 1830. Há hipoestesia facial que se estende da órbita ao longo da asa do nariz à ponta do nariz e lábio superior.

O nervo mandibular (V3) é mais comumente lesado em fraturas do ramo horizontal da mandíbula. Os nervos alveolares inferiores (forame mentoniano) inervam o lábio inferior e dentes da arcada inferior. Sua lesão provoca parestesias e dor neuropática nessas regiões.

O tronco principal do trigêmeo é raramente lesado em traumas fechados, porém mais comumente afetado em traumas penetrantes (projéteis de arma de fogo) ou fraturas da fossa média e da base do crânio.

No paciente consciente a lesão do nervo é facilmente detectável e consta principalmente de lesões sensitivas (anestesia e parestesia) de suas divisões. O envolvimento motor manifesta-se por fraqueza na musculatura mastigatória.

Geralmente, a lesão sensitiva é parcial e de leve intensidade e a recuperação ocorre após alguns meses.
Dor neuropática pode aparecer, assim como parestesias nas regiões inervadas pelo $\mathrm{V}$.

\section{e) Nervo facial}

A paralisia facial pode ocorrer em diferentes tipos de trauma craniano, podendo ocorrer imediatamente após o trauma ou tardiamente (dias a semanas). ${ }^{48,49}$ Classificamos as lesões do nervo facial segundo a Escala de House-Brackmann.

Devido ao seu longo e sinuoso trajeto no interior do osso temporal, o nervo facial é vulnerável ao trauma craniano penetrante ou não penetrante, assim como ao trauma cirúrgico ${ }^{50}$ nas mastoidectomias, estapedectomias, remoção de neoplasias da parótida e schwannomas vestibulares. Traumas que envolvam a orelha média geralmente lesam o segmento horizontal do nervo. A laceração do nervo facial no interior do canal auditivo interno ocorre em aproximadamente 50\% dos casos $^{2}$ com fraturas transversas do osso temporal. A maior parte das fraturas horizontais do temporal não atravessa o conduto auditivo interno, mas pode lesar a porção timpânica ou mastoide do nervo. Nesses casos, a paralisia facial é tardia e devida ao edema traumático.

$\mathrm{O}$ nervo facial é lesado mais frequentemente por fraturas temporais decorrentes de acidentes automobilísticos ou traumas penetrantes e não penetrantes. ${ }^{51-53}$

As fraturas temporais são classificadas como longitudinais e transversas em relação ao eixo maior do osso temporal..$^{54}$ Setenta a $80 \%$ das fraturas temporais são longitudinais e $10 \%$ a $30 \%$ são transversais. ${ }^{55,56}$ Aproximadamente $10 \%$ são do tipo misto (combinação entre longitudinal e transversa).

Nas fraturas longitudinais, o nervo é tipicamente lesado na região de transição do segmento timpânico para o mastoide (10\% a $20 \%)$. A fratura corre nas porções anterior e posterior da porção petrosa do temporal e segue para o canal falopiano através do tegmen tympani anteriormente à cápsula ótica para terminar na fossa craniana média nas proximidades do forame espinhoso (fratura longitudinal anterior). $\mathrm{Na}$ fratura longitudinal posterior, a fratura inicia-se no osso parietal e estende-se através das células aéreas mastoides, porção superoposterior do meato acústico externo, tegmen tympani e cápsula ótica para terminar na fossa média. Pode haver contusão do nervo ou lesão por fragmentos ósseos. Geralmente ocorre laceração na pele do meato acústico externo e da membrana timpânica. A perda auditiva geralmente é do tipo condutiva e não há evidência de lesão vestibular. Otorreia é frequente e causada por laceração dural no tegmen tympani. A lesão do nervo é tardia e o gânglio geniculado é a região do nervo mais frequentemente afetada. Ocorre hematoma intraneural em 30\% dos casos, rotura do nervo em 30\% 
e presença de fragmento ósseo comprimindo o nervo em $20 \%$. O sinal de Battle pode ser visto. ${ }^{57}$

Nas fraturas transversas, o nervo é frequentemente lacerado, muitas vezes com lesão concomitante de VIII nervo. Seguem-se a traumas frontais ou occipitais, geralmente iniciando no forame magno, e estendem-se perpendicularmente ao longo eixo do osso temporal, fraturando a cápsula ótica e terminando na fossa craniana média. A pele do meato acústico externo e a da membrana timpânica estão intactas. Hemotímpano é usualmente presente. Existem perda auditiva neurossensorial e evidência clínica de trauma vestibular (náuseas e vertigem). A paralisia facial ocorre em $40 \%$ a $50 \%$ e geralmente é de aparecimento imediato após a lesão por avulsão ou laceração do nervo por fragmento ósseo. O gânglio geniculado é a parte do nervo mais frequentemente acometida. ${ }^{57}$

Por meio da propedêutica, pode-se determinar qual segmento do nervo está lesado (diagnóstico topográfico). Lesões do segmento mastoide vertical resultam em perda gustativa nos dois terços anteriores da língua. Lesões que envolvem o segmento horizontal do nervo no interior da orelha média produzem a perda do reflexo estapediano (hipersensibilidade aos sons de alta frequência) e perda gustativa ipsilateral. Finalmente, lesões nos segmentos labirínticos proximais resultam em diminuição do lacrimejamento, perda do reflexo estapediano e gustação ipsilaterais à lesão.

A lesão do facial ocorre por tração, compressão ou laceração do nervo. Quando o aparecimento da paralisia é imediata após o trauma, sugere-se lesão direta ao nervo, com prognóstico funcional pior. ${ }^{58}$

A paralisia tardia geralmente decorre de pressão exercida sobre o nervo por hematoma, edema ou mesmo tecido de granulação no interior do canal falopiano. ${ }^{59}$

A paralisia facial pode ser detectada no paciente inconsciente mediante a aplicação de estímulos dolorosos e observação da movimentação facial.

A otoscopia pode revelar hemotímpano, deformidade do canal (sugerindo fratura do rochedo) e perfuração da membrana timpânica.

Da mesma forma que na lesão de nervo óptico, Patel et al. ${ }^{1}$ preconizam o uso de corticoides para a lesão do facial, porém em doses menores quando comparadas à lesão do nervo óptico, observando-se melhora na função do facial após alguns meses, principalmente nos pacientes que desenvolveram paralisia facial tardia.

A tomografia computadorizada de crânio de alta resolução com janela óssea é o procedimento de escolha para a avaliação do trauma temporal e base de crânio. ${ }^{60}$

A paralisia facial tardia ocorre em média entre $2 \mathrm{e}$ 10 dias após o trauma ${ }^{4}$ e a maioria (90\%) está associada à fratura da calvária que se dirige à base do crânio e à orelha média. A fratura também pode direcionar-se para a escama do osso temporal e atingir o tegmen tympani cruzando o nervo facial em sua porção vertical ou nas proximidades do gânglio geniculado.

Para Fisch, ${ }^{61}$ a eletroneuronografia é essencial para a indicação cirúrgica e prognóstico de recuperação funcional do nervo, pois, mediante registros de superfície, faz-se estimativa da população neuronal funcionalmente ativa.

Estudando a distribuição de lesão por segmentos do nervo facial, esse autor apresenta o seguinte achado: 93\% no segmento labiríntico e $7 \%$ no segmento timpânico.

Entre as causas de lesão, cita a presença de fragmentos ósseos exercendo pressão sobre o nervo, perda total da continuidade do nervo e presença de hematomas intraneurais.

Além do nervo facial, as fraturas temporais podem acometer o nervo trigêmeo e abducente, porém mais raramente, ${ }^{46}$ assim como trombose de seio sigmoide e fístula carótido-cavernosa.

Hagan et al..$^{62}$ descrevem lesões traumáticas específicas do osso temporal e do nervo facial quando causadas por projéteis de arma de fogo.

Em sua casuística, $43 \%$ dos casos apresentaram projéteis de arma de fogo que transfixaram o osso temporal. Em virtude de sua estrutura compacta, ocorre resistência à progressão do projétil e, quando penetrante, o próprio parênquima cerebral oferece resistência à progressão maior. Consequentemente, lesões intracranianas estão presentes por causa da natureza cinética do projétil.

A trajetória do projétil é predominantemente tangencial ao eixo maior do temporal. Logicamente, o trajeto longitudinal ao maior eixo do osso é traduzido por maior gravidade e mortalidade. ${ }^{63}$

O meato acústico externo é frequentemente lesado devido à sua localização central. A energia cinética no impacto leva à transmissão de ondas de choque para a porção cartilaginosa do meato. $O$ anel ósseo timpânico pode resistir temporariamente ao impacto ou romper devido a fragmentos metálicos. A desvitalização das lacunas ósseas leva à necrose e à sequestração. A pele macerada com obstrução por edema permite que a flora bacteriana local leve à otite externa.

\section{f) Nervo vestíbulo-coclear}

A lesão do VIII nervo é comumente vista nas fraturas que envolvem a porção petrosa do osso temporal, sejam elas longitudinais ou transversas. ${ }^{64}$

Pode ocorrer surdez por trauma envolvendo a orelha média. ${ }^{65}$ Trata-se geralmente de trauma com alta energia cinética e frequentemente associa-se à fístula liquórica, por laceração da dura-máter. A surdez ocorre pela presença de sangue ou liquor na orelha média, rotura da membrana timpânica, deslocamento da cadeia ossicular ou do tensor do tímpano e lesão da tuba de Eustáquio. ${ }^{4}$ 
À inspeção, nota-se a presença de otorragia, que pode ser importante causa de surdez de condução.

$\mathrm{Na}$ série de Patel et al., ${ }^{1}$ as lesões do VIII nervo apresentaram-se preferencialmente com surdez neurossensitiva, seguida pela condutiva e mista.

Segundo esse autor, vertigem e nistagmo associamse a fraturas temporais transversas e são sintomas resistentes ao tratamento medicamentoso.

Fraturas do osso temporal geralmente resultam de trauma direto ao crânio na região parietal. Como a cápsula ótica, que envolve a orelha interna, é formada por tecido ósseo denso, a fratura cursa ao redor dessa estrutura e lesa os forames da base do crânio, como o forame jugular.

Há fraturas que envolvem as proximidades do meato acústico externo e dirigem-se ao maior eixo da porção petrosa do osso temporal, estendendo-se anteriormente ao forame lácero e à artéria carótida interna. ${ }^{2}$

Concussão labiríntica é definida como a presença de sintomas auditivos e vestibulares (isolados ou em combinação) que resultam de trauma craniano sem a presença de fraturas temporais. Embora estejam protegidas por uma cápsula óssea, as membranas labirínticas são suscetíveis ao trauma não penetrante..$^{66}$

A vertigem posicional pós-traumática é a sequela neuro-otológica mais comum. O paciente desenvolve ataques de vertigem curtos e súbitos associados ao nistagmo e precipitados por mudanças na posição da cabeça (movimentos de flexão, extensão e rotação da cabeça). A vertigem dura menos de 1 minuto, mas tonturas com náuseas podem ser persistentes. Acredita-se que seja causada quando os cristais de carbonato de cálcio $\left(\mathrm{CaCO}_{3}\right)$ da mácula do utrículo desloquem-se para o canal semicircular posterior.

Fístula perilinfática traumática ocorre quando existe lesão das membranas que limitam o labirinto. Deve ser suspeitada quando o paciente apresentar início súbito de perda auditiva associada a vertigem e tinnitus imediatamente após trauma craniano. Muitos pacientes referem ouvir um estampido após o trauma seguido de perda auditiva, tinnitus e vertigem. ${ }^{64}$

\section{Conclusões}

Os nervos olfatório, oculomotor e facial foram os nervos mais frequentemente lesados, seguidos pelo óptico e vestíbulo-coclear.

A maioria dos pacientes com lesão de nervo olfatório não apresentou lesão intracraniana; são pacientes que evoluíram relativamente bem quanto à recuperação da função olfativa, mas apresentaram excelente recuperação em termos de atividade da vida diária; a maior parte das lesões do olfatório ocorre de forma isolada; em termos de etiologia, todas sugeriram mecanismos de desaceleração repentina.

A lesão do nervo óptico geralmente ocorre associada a lesões de outros nervos, sendo o acidente motociclístico a causa principal.

A maior parte dos pacientes com lesão de nervo óptico apresentava nível de consciência suficiente para relatar queixa de baixa acuidade visual pós-traumática.

A lesão do nervo oculomotor é frequente (seis casos) e associada a lesão de outros nervos cranianos e lesões intracranianas; etiologias traumáticas de alta energia cinéticas (acidente motociclístico) foram as principais causas de lesão do oculomotor; sua lesão pode ser detectada independentemente do nível de consciência do paciente.

O nervo troclear raramente é lesado isoladamente como decorrência de etiologia traumática; quando ocorre, a fratura frontal está presente com ausência de lesões intracranianas.

O nervo abducente geralmente é lesado conjuntamente com outros nervos; a maior parte desses pacientes apresentava lesões intracranianas associadas e fraturas demonstráveis; a totalidade dos pacientes evoluiu excelentemente para assumir as atividades da vida diária.

A lesão do nervo trigêmeo é rara e nos casos descritos ocorreu exclusivamente em associação à lesão de outros nervos; a maior parte dos pacientes apresentou fraturas de teto orbitário e lesões intracranianas associadas.

As lesões dos nervos facial e do vestíbulo-coclear estão relacionadas, geralmente, a fraturas e lesões intracranianas.

\section{Agradecimentos}

Às disciplinas de Oftalmologia, Otorrinolaringologia e ao Serviço de Fonoaudiologia da Santa Casa de Misericórdia de São Paulo.

Aos residentes da disciplina de Neurocirurgia da Santa Casa de Misericórdia de São Paulo.

\section{Referências}

1. Patel P, Kalyanaraman S, Reginald J, Natarajan P, Ganapathy K, Suresh Bapu KR, et al. Post-traumatic cranial nerve injury. Indian J Neurotrauma. 2005;2(1):27-32.

2. Keane JR, Baloh RW. Post-traumatic neuropathies. In: Evans RW, editor. The neurology of trauma. Philadelphia: Saunders; 1996; p. 117-32. 
3. Leigh AD. Defects of smell after head injury. Lancet. 1943;244:38-40.

4. Hughes B. The results of injury to special parts of the brain and skull: the cranial nerves. In: Rowbotham GF, editor. Acute injuries of the head. Baltimore: Williams \& Wilkins; 1964. p. 408-10.

5. Sumner D. Post-traumatic anosmia. Brain. 1964;87:107-20.

6. Hagan PJ. Posttraumatic anosmia. Arch Otolaryngol. 1967;85(1):85-9.

7. Jafek BW, Eller PM, Esses BA, Moran DT. Post-traumatic anosmia. Ultrastructural correlates. Arch Neurol. 1989;46(3):300-4.

8. Levin HS, High WM, Eisenberg HM. Impairment of olfactory recognition after closed head injury. Brain. 1985;108 (Pt 3):579-91.

9. Schechter PJ, Henkin RI. Abnormalities of taste and smell after head trauma. J Neurol Neurosurg Psychiatry. 1974;37(7):802-10.

10. Griffith IP. Abnormalities of smell and taste. Practitioner. 1976;217(1302):907-13.

11. Sumner D. Disturbance of senses of smell and taste after head injuries. In: Vinken PJ, Bruyn BW, editors. Handbook of clinical neurology. Amsterdam: Elsevier; 1976. p. 1-25.

12. Turner JWA. Indirect injuries of the optic nerve. Brain. 1943;66:140-51.

13. Kline LB, Morawetz RB, Swaid SN. Indirect injury of the optic nerve. Neurosurgery. 1984;4(6):756-64.

14. Crompton MR. Visual lesions in closed head injury. Brain. 1970;93(4):785-92.

15. Pringle $\mathrm{JH}$. Monocular blindness following diffused violence to the skull: its causation and treatment. $\mathrm{Br} \mathrm{J}$ Surg. 1916;4:373-85.

16. Joseph MP, Lessell S, Rizzo J, Momose KJ. Extracranial optic nerve decompression for traumatic optic neuropathy. Arch Ophthalmol. 1990;108(8):1091-3.

17. Hughes B. Indirect injury of the optic nerves and chiasma. Bull Johns Hopkins Hosp. 1962;111:98-126.

18. Noble MJ, McFadzean R. Indirect injury to the optic nerves and optic chiasm. Neuroophthalmology. 1987;7(6):341-8.

19. Edmund J, Godtfredsen E. Unilateral optic atrophy following head injury. Acta Ophthalmol (Copenh). 1963;41:693-7.

20. Spoor TC, Hartel WC, Lensink DB, Wilkinson MJ. Treatment of traumatic optic neuropathy with corticosteroids. Am J Ophthalmol. 1990;110(6):665-9.

21. Lam BL, Weingeist TA. Corticosteroid-responsive traumatic optic neuropathy. Am J Ophthalmol. 1990;109(1):99-101.

22. Braughler JM, Hall ED. Current application of "highdose" steroid therapy for CNS injury. A pharmacological perspective. J Neurosurg. 1985;62(6):806-10.

23. Miller NR. The management of traumatic optic neuropathy. Arch Ophthalmol. 1990;108(8):1086-7.

24. De Vries-Knoppert WA. Evulsion of the optic nerve. Doc Ophthalmol. 1989;72(3-4):241-5.

25. Hedges TR 3rd, Gragoudas ES. Traumatic anterior ischemic optic neuropathy. Ann Ophthalmol. 1981;13(5):625-8.

26. Wyllie AM, McLeod D, Cullen JF. Traumatic ischaemic optic neuropathy. Br J Ophthalmol. 1972;56(11):851-3.

27. Guy J, Sherwood M, Day AL. Surgical treatment of progressive visual loss in traumatic optic neuropathy. Report of two cases. J Neurosurg. 1989;70(5):799-801.

28. Rucker CW. Paralysis of the third, fourth and sixth cranial nerves. Am J Ophthalmol. 1958;46(6):787-94.

29. Rush JA, Younge BR. Paralysis of cranial nerves III, IV, and VI. Cause and prognosis in 1000 cases. Arch Ophthalmol. 1981;99(1):76-9.
30. Harley RD. Paralytic strabismus in children. Etiologic incidence and management of the third, fourth, and sixth nerve palsies. Ophthalmology. 1980;87(1):24-43.

31. Miller NR. Solitary oculomotor nerve palsy in childhood. Am J Ophthalmol. 1977;83(1):106-11.

32. Green WR, Hackett ER, Schlezinger NS. Neuroophthalmologic evaluation of oculomotor nerve paralysis. Arch Ophthalmol. 1964;72:154-67.

33. Memon MY, Paine KW. Direct injury of the oculomotor nerve in craniocerebral trauma. J Neurosurg. 1971;35(4):461-4.

34. Chapman LI, Urist MJ, Folk ER, Miller MT. Acquired bilateral superior oblique muscle palsy. Arch Ophthalmol. 1970;84(2):137-42.

35. Younge BR, Sutula F. Analysis of trochlear nerve palsies. Diagnosis, etiology, and treatment. Mayo Clin Proc. 1977;52(1):11-8.

36. Khawam E, Scott AB, Jampolsky A. Acquired superior oblique palsy. Diagnosis and management. Arch Ophthalmol. 1967;77(6):761-8.

37. Burger LJ, Kalvin NH, Smith JL. Acquired lesions of the fourth cranial nerve. Brain. 1970;93(3):567-74.

38. Lee J, Flynn JT. Bilateral superior oblique palsies. Br J Ophthalmol. 1985;69(7):508-13.

39. Sydnor CF, Seaber JH, Buckley EG. Traumatic superior oblique palsies. Ophthalmology. 1982;89(2):134-8.

40. Keane JR. Bilateral sixth nerve palsy. Analysis of 125 cases. Arch Neurol. 1976;33(10):681-3.

41. Schneider RC, Johnson FD. Bilateral traumatic abducens palsy. A mechanism of injury suggested by the study of associated cervical spine fractures. J Neurosurg. 1971;34(1):33-7.

42. Arias MJ. Bilateral traumatic abducens nerve palsy without skull fracture and with cervical spine fracture: case report and review of the literature. Neurosurgery. 1985;16(2):232-4.

43. Shrader EC, Schlezinger NS. Neuro-ophthalmologic evaluation of abducens nerve paralysis. Arch Ophthalmol. 1960;63:84-91.

44. Rucker CW. The causes of paralysis of the third, fourth and sixth cranialnerves. Am J Ophthalmol. 1966;61(5 Pt 2):1293-8.

45. Jefferson G, Schorstein J. Injuries of the trigeminal nerve, its ganglion and its divisions. Br J Surg. 1955;42(176):561-81.

46. Ghorayeb BY, Yeakley JW, Hall JW 3rd, Jones BE. Unusual complications of temporal bone fractures. Arch Otolaryngol Head Neck Surg. 1987;113(7):749-53.

47. Russell W. Brock's injury of the brain and spinal cord. 3rd ed. Baltimore: Williams and Wilkins; 1949.

48. Potter JM. Facial palsy following head injury. J Laryngol Otol. 1964;78:654-7.

49. May M. Trauma to the facial nerve. Otolaryngol Clin North Am. 1983;16(3):661-70.

50. Miehlke A. Typical sites of facial-nerve lesions. Arch Otolaryngol. 1969;89(1):122-6.

51. Cannon CR, Jahrsdoerfer RA. Temporal bone fractures. Review of 90 cases. Arch Otolaryngol. 1983;109(5):285-8.

52. Lindeman RC. Temporal bone trauma and facial paralysis. Otolaryngol Clin North Am. 1979;12(2):403-13.

53. Harker LA, McCabe BF. Temporal bone fractures and facial nerve injury. Otolaryngol Clin North Am. 1974;7(2):425-31.

54. Goodwin WJ Jr. Temporal bone fractures. Otolaryngol Clin North Am. 1983;16(3):651-9.

55. Lambert PR, Brackmann DE. Facial paralysis in longitudinal temporal bone fractures: a review of 26 cases. Laryngoscope. 1984;94(8):1022-6. 
56. Adkins WY, Osguthorpe JD. Management of trauma of the facial nerve. Otolaryngol Clin North Am. 1991;24(3):587-611.

57. Schubiger O, Valavanis A, Stuckmann G, Antonucci F. Temporal bone fractures and their complications. Examination with high resolution CT. Neuroradiology. 1986;28(2):93-9.

58. Adour KK, Boyajian JA, Kahn ZM, Schneider GS. Surgical and nonsurgical management of facial paralysis following closed head injury. Laryngoscope. 1977;87(3):380-90.

59. Adegbite AB, Khan MI, Tan L. Predicting recovery of facial nerve function following injury from a basilar skull fracture. J Neurosurg. 1991;75(5):759-62.

60. Aguilar EA 3rd, Yeakley JW, Ghorayeb BY, Hauser M, Cabrera J, Jahrsdoerfer RA. High resolution CT scan of temporal bone fractures: association of facial nerve paralysis with temporal bone fractures. Head Neck Surg. 1987;9(3):162-6.

61. Fisch U. Facial paralysis in fractures of the petrous bone. Laryngoscope. 1974;84(12):2141-54.

62. Hagan WE, Tabb HG, Cox RH, Travis LW. Gunshot injury to the temporal bone: an analysis of thirty-five cases. Laryngoscope. 1979;89(8):1258-72.
63. Duncan NO 3rd, Coker NJ, Jenkins HA, Canalis RF. Gunshot injuries of the temporal bone. Otolaryngol Head Neck Surg. 1986;94(1):47-55

64. Griffiths MV. The incidence of auditory and vestibular concussion following minor head injury. J Laryngol Otol. 1979;93(3):253-65.

65. Browning GG, Swan IR, Gatehouse S. Hearing loss in minor head injury. Arch Otolaryngol. 1982;108(8):474-7.

66. Schuknecht HF, Neff WD, Perlman HB. An experimental study of auditory damage following blows to the head. Ann Otol Rhinol Laryngol. 1951;60(2):273-89.

\section{Endereço para correspondência}

Luiz Fernando Cannoni

Rua Conselheiro Cotegipe, 543, Belenzinho

03058-000 - São Paulo, SP, Brasil

Telefone: (11) 2796-7113

E-mail: luiz2603@terra.com.br 